\title{
ANALYSIS AND PREDICTION OF THE EARING BEHAVIOUR OF LOW CARBON STEEL SHEET
}

\author{
RAFAEL SCHOUWENAARS*, PAUL VAN HOUTTE*, \\ ALBERT VAN BAEL*, JAN WINTERS* and KOEN MOLS** \\ *Department of Metallurgy and Materials Engineering, \\ Katholieke Universiteit Leuven, B-3001 Leuven, Belgium, \\ **OCAS NV, John Kennedylaan, 3, B-9060 Zelzate, Belgium
}

(Received 3 July 1995)

\begin{abstract}
The earing behaviour during a cup drawing test was studied for a wide range of low carbon steel sheets, in order to check the validity of an existing model for the earing of anisotropic metal sheets. The modelling of the anisotropic behaviour of the metal sheet was made using the Taylor theory of polycrystal plasticity, which was implemented in a simplified cinematic model for the material flow during a cup-drawing test.

Both the relaxed constraints and full constraints model were tested and mutually compared. This evaluation was one of the main goals of the research.

It will be shown that by using the full constraints Taylor theory, a very good correlation can be found between measured and predicted ear heights after cup drawing. Deviations of the predicted behaviour can be explained on the basis of literature data about the texture evolution during a cup drawing test. The model could however not predict the average cup height accurately. This is probably due to the fact, that the model does not take the ironing effect that takes place during the cup drawing test into account.
\end{abstract}

KEY WORDS: Cup drawing, earing, full constraints Taylor theory, relaxed constraints Taylor theory.

\section{INTRODUCTION}

The cup-drawing test is a mechanical testing method in which the deep-drawability of a material is characterised. Circular blanks are deep drawn to the form of cylindrical cups by means of a classical tool geometry consisting of a cylindrical punch, a die and a blank holder. The deep drawability of the material is characterised by the ratio of the diameter of the blank at which failure starts to occur during the test to the punch diameter. This so called "Limiting Drawability Ratio" (LDR) is a good characteristic of the deep-drawability of a sheet metal and is strongly correlated to the $R$-value of the material. This latter value is defined as the ratio between the true plastic strain in width direction and the true plastic strain in thickness direction during a uniaxial tensile test.

One of the well known phenomena occurring during a cup-drawing test is the so called earing. It is observed that the height of the drawn cup depends on the position at which it is measured. As the test and sample geometry are perfectly axisymmetric, the reason for earing must be attributed to the anisotropy of the material. In low (LC) 
to ultra low carbon (ULC) steel sheets, this anisotropy can almost completely be explained from the crystallographic texture of the sheet metal.

The earing behaviour of sheet metals is extensively studied for aluminium alloys for packaging, as the phenomenon lies at the base of considerable material losses during can production. In the study of steel sheets for automotive applications, the earing behaviour is considered less important. However, it is clear that the directionally inhomogeneous deformation behaviour of the material during deep drawing, which is caused by the planar anisotropy of the sheet, can not be advantageous for the overall formability of the sheet during press forming.

Tucker (1961) presented a method to predict the earing of a single crystalline sheet. Later authors (Da Costa Viana et al., 1978; Pochettino et al., 1981; Rodriguez and Bate, 1985; Van Houtte, Cauwenbergs and Aernoudt, 1987; Lin et al., 1991; Barlat et al., 1991, Ceccaldi, 1994) presented more or less complex methods for earing prediction of anisotropic materials with a known crystallographic texture. Those will not be discussed into detail here.

The model used in this paper is the one presented by Van Houtte et al. (1993), which is a fairly simple and easy to use model. It is based on the crystallite orientation distribution function (ODF) of the material and on a yield locus derived from it by means of the Taylor-Bishop-Hill-theory. Only the basic outline of the model will be discussed here. For the details, we refer to Van Houtte, Clarke and Saimoto (1993).

\section{BASIC OUTLINE OF THE MODEL}

\section{Basic Assumptions}

In the model, it is assumed that plane stress exists in the blank. So, the stress $\sigma_{z z}$ in the thickness direction is assumed to be zero, as well as the shear stresses $\sigma_{z \psi}$ and $\sigma_{\mathrm{zr}}$ in which $r$ denotes the radial direction and $\psi$ the tangential direction. This does not imply that the strain $\varepsilon_{z z}$ be zero. So, the thickness of the flange is allowed to change during the test, as is observed experimentally.

Furthermore, radial flow is assumed. Each point of the flange moves toward the centre of the flange, with a velocity which may vary in function of the angle $\psi$ to the rolling direction of the sheet (Figure 1). This allows to calculate the velocity of the points

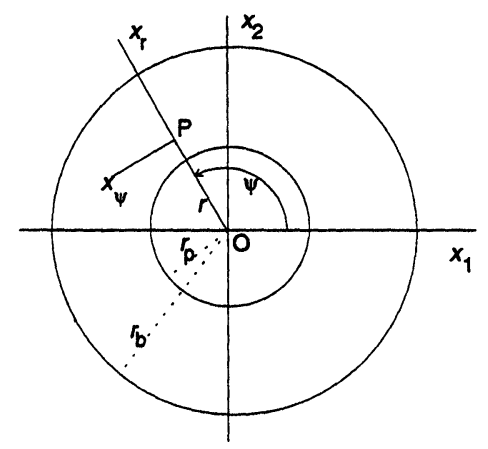

Figure 1 Definition of the reference system and the angle $\psi$ in a circular blank. $x_{1}$ is the rolling direction. 
on the outer rim of the blank as a function of time and $\psi$. In a first approximation, the cup height is equal to the time multiplied by the velocity of the punch. The cup height obtained in this way will be function of $\psi$.

\section{Implementation of Texture Influence; the Constant S-model}

As the outer rim of the blank is a free surface, the stress components $\sigma_{\mathrm{rr}}$ and $\sigma_{\mathrm{r} \psi}$ are zero, together with the aforementioned stress components which were zero under the assumption of plane stress. The only stress component which can differ from zero is the tangential stress $\sigma_{\psi \psi \psi}$, which needs to be compressive. Its position on a yield surface is presented in Figure 2. The shape of the yield locus will be calculated from texture data. For sheet metal forming, if all stresses are in the plane of the sheet, one can use a two-dimensional section through the 5-dimensional yield surface to represent all stress states in the sheet. Dependent of the test direction $\psi$, another section will have to be chosen. The shape of the yield locus section under concern will thus depend on $\psi$. According to the normality rule, the normal to the yield locus for a given stress state, is proportional to the vector representation of the strain rate. The direction of this vector depends on the shape of the yield locus, and thus on the texture. This direction can be calculated and is given by :

$$
q=-\frac{\dot{\varepsilon}_{r r}}{\dot{\varepsilon}_{\psi \psi}}
$$

$q$ is sometimes called the contraction coefficient. Its value is calculated from the texture as will be shortly described later. It is a function of $\psi$.

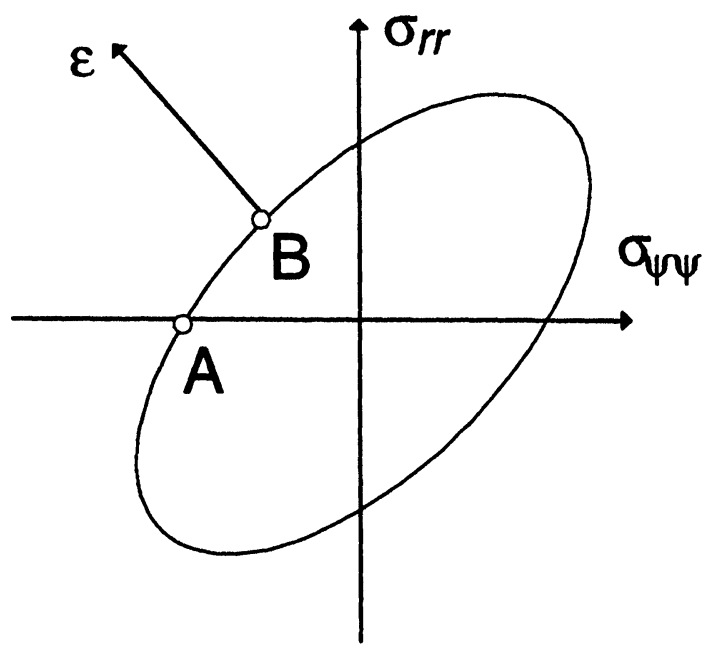

Figure 2 Representation of a yield locus section. The position of the stress state at the rim of the blank is situated in point $A$, the stress state inside the blank is indicated in B. The plastic strain is a vector normal to the yield surface at $\mathrm{B}$. The strain ratio $\mathrm{q}$ (eq. (1)) characterises its direction. 
For points on the blank which are not situated at the outer rim, the stress $\sigma_{\pi}$ is not equal to zero. In the model, the stress $\sigma_{\mathrm{r} \psi}$ will be neglected, not so the stress $\sigma_{\mathrm{rr}}$. Hence, a point $B$ on the stress surface must be considered for which the ratio of the stress ratio $s$, given by

$$
s=\frac{\sigma_{r r}}{\sigma_{\psi \psi}}
$$

is negative. The value of $q$ which corresponds to such point B does not only depend on $\psi$, but also on $s$ (Figure 2).

In order to overcome the problems of integrating the deformation in the blank between the punch radius and the blank radius at a certain time $t$ during the process for a variable value of $s$, an analysis was made in which $s$ is considered constant over the blank diameter. A value was chosen which is approximately equal to the average value over the deformed part of the blank diameter. A reasonable approximation of this average can be derived analytically starting from the assumption of a von Mises yield criterion. It was found that the value of $s$ depends almost linearly on the value of the drawing ratio (DR), which is defined as the ratio between the blank radius and the radius of the punch. This relationship is presented in Figure (3). For a more detailed analysis of this problem, we refer again to Van Houtte, Clarke and Saimoto (1993).

\section{Formulation of the Model}

For the final formulation of the model, also the thickness of the sheet and the roundoff radius of the punch are taken into account. The height of the cup in a direction $\psi$ is given by following formula (Van Houtte, Clarke and Saimoto, 1993):

$$
h(\psi)=d+\left(1-\frac{\pi}{4}\right)\left(2 r_{p p}+d\right)+\frac{r_{b}^{q+1}-\left(r_{p}+d\right)^{q+1}}{(q+1)\left(r_{p}+\frac{d}{2}\right)^{q}}
$$

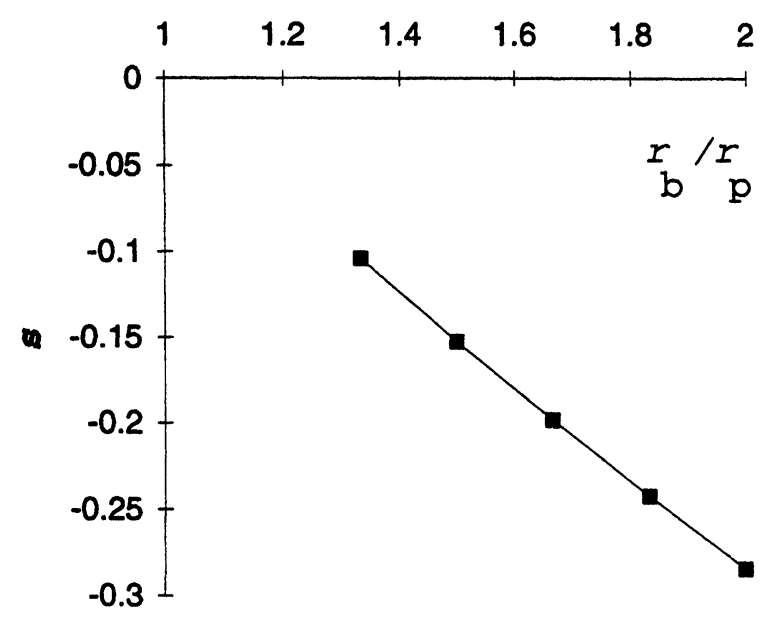

Figure 3 Relationship between the Drawing ratio and s. 
Herein, $h(\psi)$ is the height of the cup in direction $\psi$ to the rolling direction, $d$ the initial thickness of the blank, $r_{\mathrm{pp}}$ the radius of the punch profile, $r_{\mathrm{b}}$ the initial radius of the blank and $r_{\mathrm{p}}$ is the radius of the punch. As was mentioned earlier, the value $q$ must be chosen for an appropriate value of $s$.

\section{Calculation of the Yield Surface}

The yield surface was calculated using the Taylor-Bishop-Hill (TBH) theory using the method described by Van Houtte (1987) and Van Houtte et al. (1989), which is based on the series expansion of the ODF and of the Taylor factor $M$ in function of the crystallite orientation $g$.

An important point of discussion in the use of the TBH-theory for the prediction of the anisotropy of low carbon steel sheet is whether the full constraints (FC) or the relaxed constraints (RC) (Van Houtte, 1988) theory is more accurate. This discussion is treated in several papers (Daniel and Jonas 1990; Schouwenaars et al., 1994). Although it was stated in those papers that for the prediction of the $R$-value of low carbon steel sheet, the RC-theory gave best results, this question was reconsidered.

Another point of discussion is the question about which set of potential slip systems should be used in the predictions. The choice to be made is between:

1) $\{110\}<111>$

2) $\{112\}<111>$ and $\{110\}<111>$

3) $\{123\}<111>$ and $\{112\}<111>$ and $\{110\}<111>$

The latter is a close approximation of the so-called "pencil glide model", which accepts all planes as possible glide planes, as long as de slip direction is $<111>$.

Traditionally, the second set of slip systems is chosen. Preliminary tests showed that the addition of the $\{123\}<111>$ slip system had no significant influence on the results. It was also found that when the ratio $\alpha$ between the critical resolved shear stress on the $\{110\}<111>$ and $\{112\}<111>$ was varied from 1 to 0.95 , no important differences were seen. So, it was decided to use the $\{112\}<111>$ and $\{110\}<111>$ systems, with a ratio $\alpha$ equal to 1 for both the relaxed and the full constraints model.

\section{EXPERIMENTS AND RESULTS}

\section{Materials Characterisation}

In order to make a good characterisation of the deep-drawability of low carbon steel sheet in general, ten materials were selected with different carbon and manganese content and with different processing parameters. Two interstitial-free steels were tested (materials 1 and 8), along with three ULC-materials (100-300 ppm C; materials 2, 3 and 7), three LC-qualities (300-1000 ppm C; materials 4, 5 and 6) and two ULC-materials which received their last rolling passes during hot-rolling in the complete ferritic region (materials 9 and 10).

The textures of the materials were measured by XRD using a Siemens Goniometer with a copper tube and monochromator. The textures were obtained using the reflection method, measuring 4 incomplete pole figures (Van Houtte, 1984). The ODF's of the 


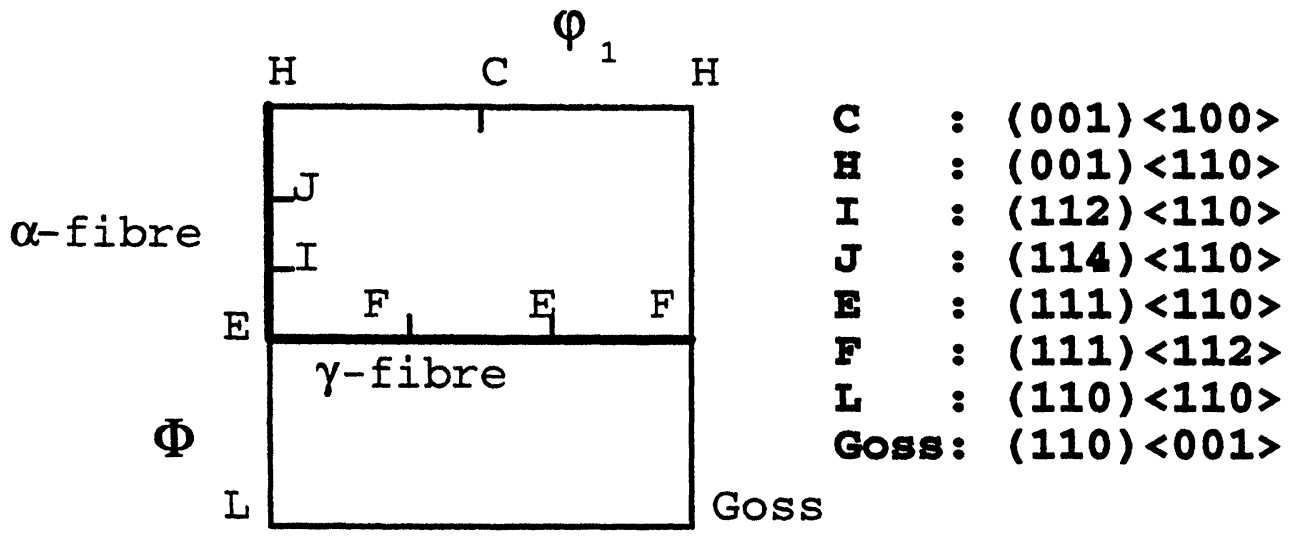

Figure 4 Important texture components of a low carbon steel sheet.

materials were calculated using the series expansion method (Bunge, 1982) with expansion coefficients up to 22 . The results of the ODF-calculation of steel can be represented by means of a $\varphi_{2}=45^{\circ}$ section, as this section contains all important recrystallisation components of a low carbon steel sheet. The main components are repeated in Figure 4, together with the symbols used to denote them further in the text.

The ODF's are, in this research, only an intermediate result, as in the end, only the value of $q$, as obtained from the yield locus for a certain value of $s$, is to be known. Therefore, not all ODF's are discussed here. However, in order to illustrate the large differences in texture between the materials, a 3-D view of the $\varphi_{2}=45^{\circ}$ sections of the ODF of four materials is given here in Figure 5 to 8 . In Figures 9 to 12 , the corresponding yield locus sections are given for a materials reference system which is rotated $0^{\circ}$ respectively $45^{\circ}$ to the rolling direction of the sheet, around the sheet normal.

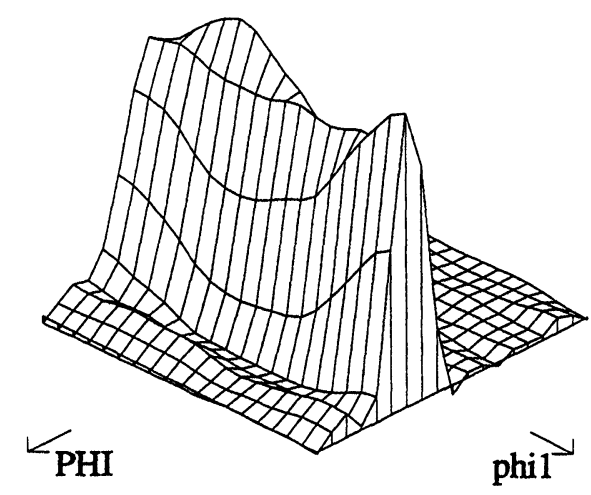

Figure $5 \varphi_{2}=45^{\circ}$ section of material 1 . A very strong $\gamma$-fibre is seen. The highest value in this figure is 13.13 . 


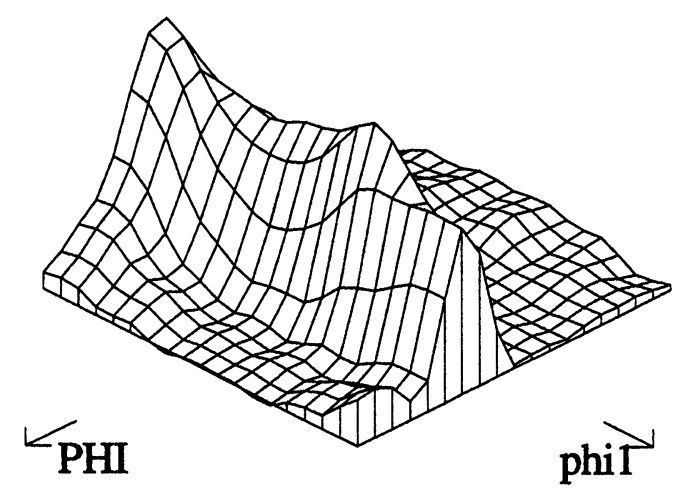

Figure $6 \varphi_{2}=45^{\circ}$ section of material 2. The $\gamma$ fibre is less intense, with a maximum on the E-component. Maximum in this figure: 8.25 .

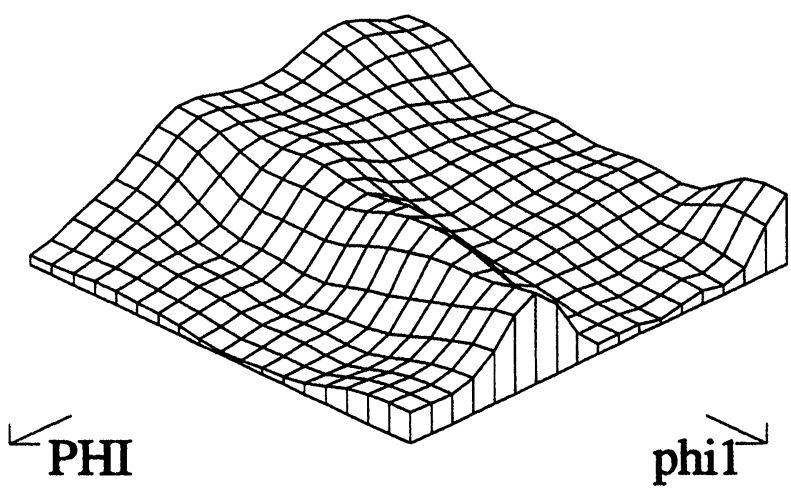

Figure $7 \varphi_{2}=45^{\circ}$ section of material 5 . There is a weak $\gamma$ fibre, together with a weak partial $\alpha$ fibre. The highest value in this figure is 3.17 .

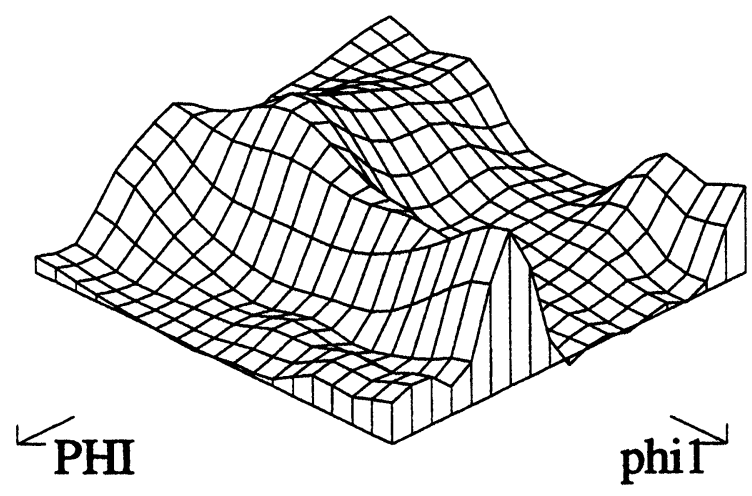

Figure $8 \varphi_{2}=45^{\circ}$ section of material 10. A weak $\gamma$ fibre can be observed together with a weak $\alpha$-fibre. maximum of this figure: 5.56 . 


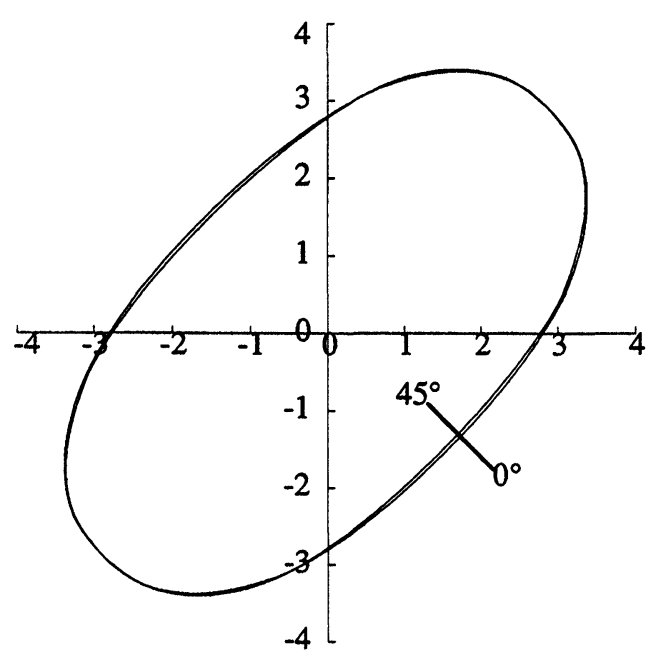

Figure 9 Yield locus of material 1.

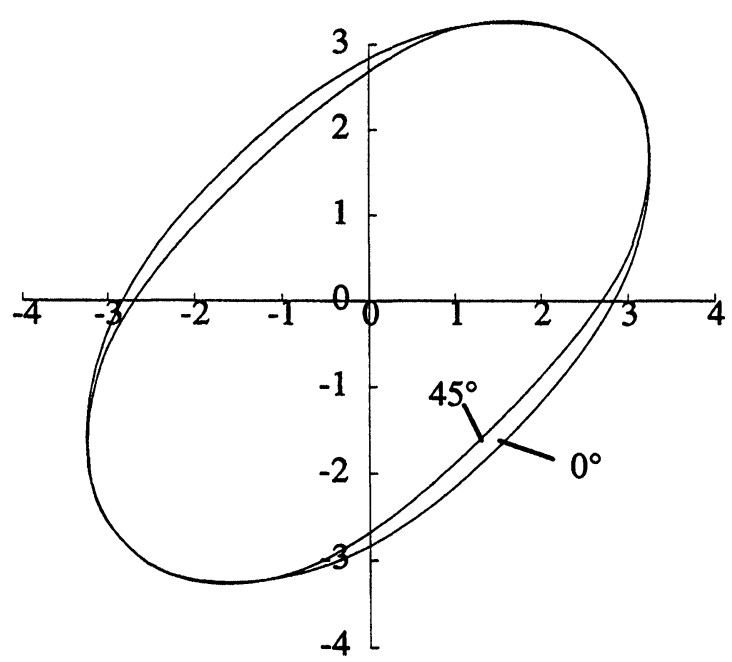

Figure 10 Yield locus of material 2.

\section{Deep Drawing Tests}

The deep drawing tests were performed on a hydraulic press, using a punch diameter of $50 \mathrm{~mm}$. The rounding of the punch had a radius of $5 \mathrm{~mm}$, those of the die 10 $\mathrm{mm}$. A blankholder force $5 \mathrm{kN}$ was applied for all tests. The tests were performed on circular blanks with a diameter between 100 and $110 \mathrm{~mm}$, except for material 1 , which had to be tested between 110 and $120 \mathrm{~mm}$. The samples were not lubricated, but no special degreasing treatment was applied either. The LDR of the different materials are listed in Table 1. The LDR of material 7 could not be measured, as its thickness was twice as large as those of the other materials. 


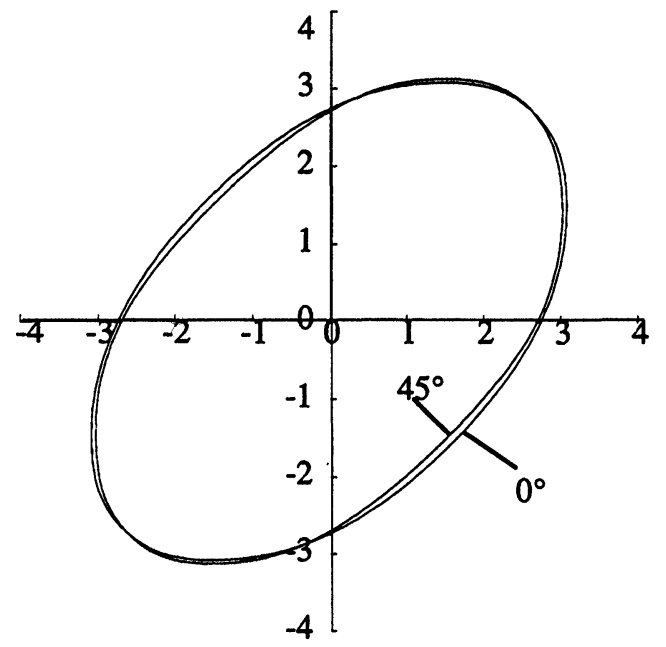

Figure 11 Yield locus of material 5.

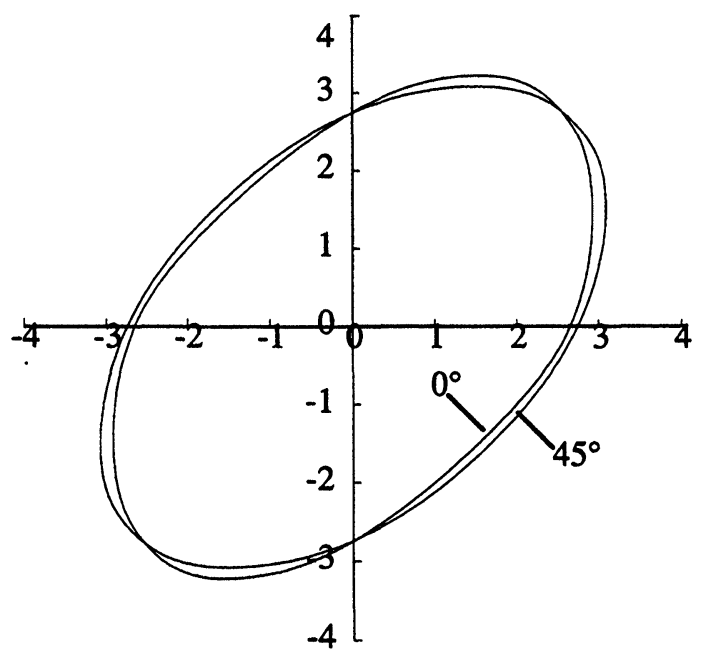

Figure 12 Yield locus of material 10.

Table 1 Limiting drawability ratio of the ten materials

\begin{tabular}{lccccccccc}
\hline Material & 1 & 2 & 3 & 4 & 5 & 6 & 8 & 9 & 10 \\
\hline LDR & 2.34 & 2.22 & 2.12 & 2.13 & 2.12 & 2.16 & 2.21 & 2.17 & 2.08 \\
\hline
\end{tabular}


The ear heights were measured on those cups which after deep drawing showed the best symmetry. This means that the ears at opposite sides of the cups have the same height, which can be obtained by a perfect centring of the circular blanks in the press before drawing. The ear heights were measured manually. Measurements were performed each $15^{\circ}$ starting from the rolling direction. As orthorhombic sample symmetry could be expected, the measurements were averaged over the symmetrically equivalent positions of the cup. The accuracy of this procedure was estimated to be $0.2 \mathrm{~mm}$.

As an example, the ear profiles of the four materials mentioned earlier are shown in Figures 13 to 16 . Those figures represent a general overview of the earing behaviour of the materials. Material 1 shows a 6-ear behaviour, with ears at $0^{\circ}$ and $75^{\circ}$, a deep trough at $30^{\circ}$ and a small trough at $90^{\circ}$. Material 2 is a classical example of 4-ear behaviour. It has the most pronounced earing of all materials studied. The ears are about the same at 0 and $90^{\circ}$, the minimum is exactly at $45^{\circ}$. This latter holds also for materials 5 and 7. However, material 5 is highly isotropic and therefore shows the smallest ears of all ten materials. Materials 4, 6, 8, 9 and 10 also have four-eared cups, but their form is less symmetric. Materials 4, 9 and 10 have higher ears at $90^{\circ}$, the latter two nearly showing a 2-ear behaviour. (with ears at 90 and $270^{\circ}$ and troughs at 0 and $180^{\circ}$ ), while 6 and 8 have higher ears at $0^{\circ}$.

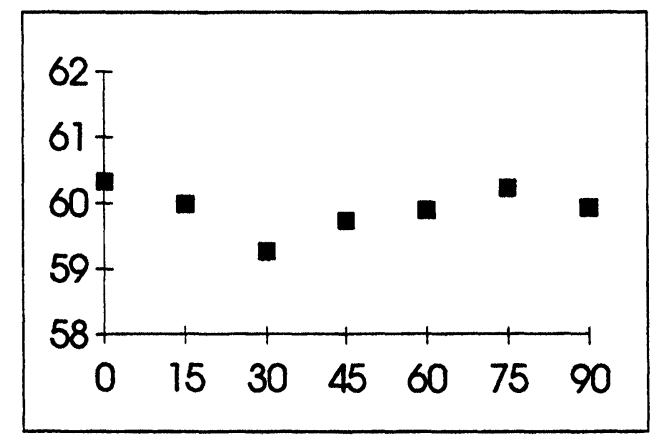

Figure 13 Cup profile of material 1.

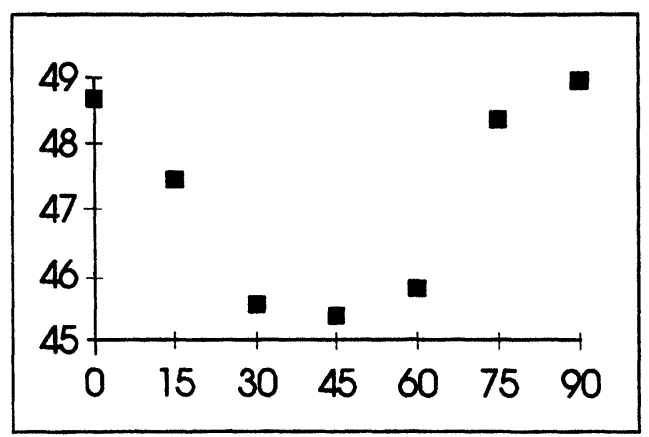

Figure 14 Cup profile of material 2. 


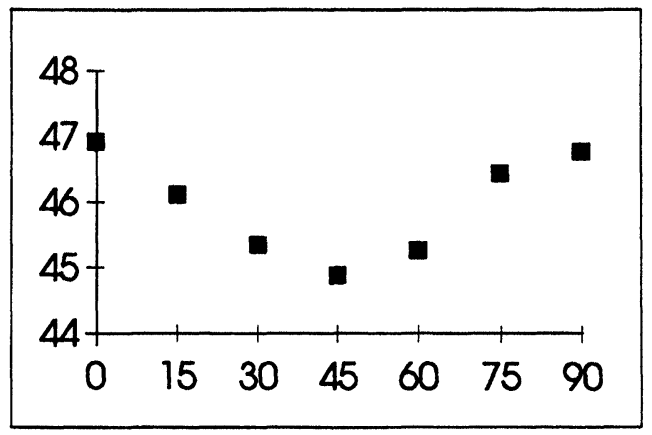

Figure 15 Cup profile of material 5.

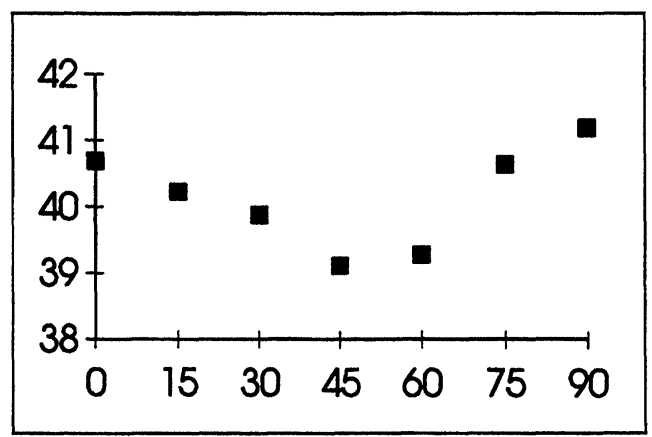

Figure 16 Cup profile of material 10.

\section{IMPORTANCE OF THE STUDY OF THE 6-EAR BEHAVIOUR IN MATERIAL 1}

The study of the earing behaviour can be used to discriminate between the accuracy of the different plasticity models which are used for the prediction of the $q$-values. This is especially true when the ears at 0 and $90^{\circ}$ are significantly different. Also the 6 -ear behaviour is very interesting for the validation of the plasticity model.

In order to get an insight in the influence of different texture components on the directional variation of $q$, several single component textures were generated by superimposing a gaussian peak upon the ideal orientation of the component under concern. The spread of this gaussian function was taken $16.5^{\circ}$. The $q$-values were calculated of the ideal textures generated in this way were calculated as function of $\psi$, using either relaxed constraints or full constraints. For the E-component $(\{111\}$ $<110>$ ), the results are illustrated in Figure 17. The $q$-values presented here are the values as would be obtained from a uniaxial tensile test, so at a value of $s$ equal to zero. Note that the tensile axis of this hypothetical test is at an angle $\psi-\pi / 2$, since $\mathrm{x}_{\psi}$ is the tangential axis at the point $\psi$ along the edge of the blank (Figure 1).

From Figure 17, it becomes clear that if one can find a material with only a strong E-component, a six-ear behaviour will occur. As the maxima of the ears will occur 


\section{E -component}

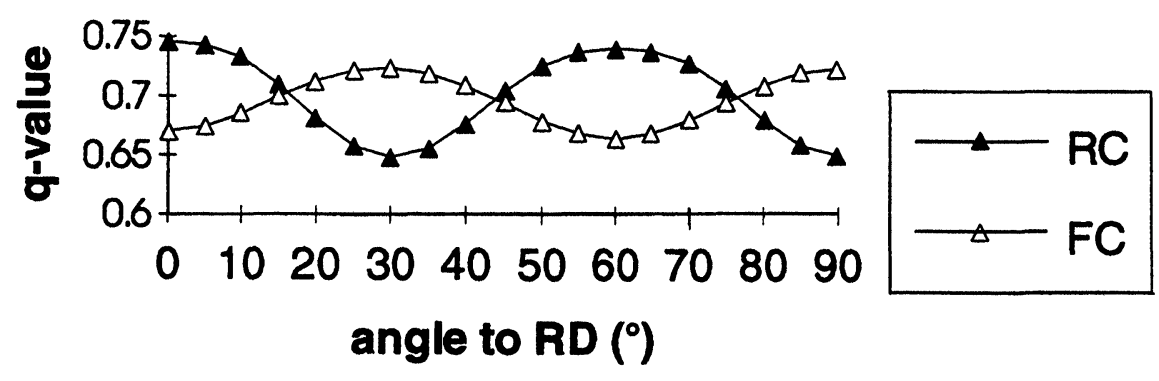

Figure 17 Calculated q-values for an ideal E component texture.

at an angle $90^{\circ}$ rotated to the maximum of the $q$-value, it is clear that for such a material the ears will lie at 0 and $60^{\circ}$ if the relaxed constraints model were more accurate, and at 30 and $90^{\circ}$ if the full constraints model would be better. Also a material with a very strong F-component texture would give rise to 6-ear behaviour, however, the ears would now be rotated $90^{\circ}$ relative to those caused by the E-component.

It might seem strange that the only material with an expressed 6-ear behaviour, namely material 1 , has a very strong $\gamma$-fibre texture, with about equal intensities at the $\mathrm{E}$ and F components. So, no earing is expected at all, as both components would cancel each other out. However, it can be found in literature (Daniel et al., 1993) that during a cup drawing process of an IF-steel, a texture evolution occurs by which the texture in the flange evolves toward the E-component. This evolution has an influence on the earing. It will be seen further on that the prediction of the ear height for material 1 should take this texture evolution into account.

\section{PREDICTION OF THE EAR HEIGHT}

In this section, the predicted ear height will be compared to the measured values. Not all ten materials will be discussed into detail, although the calculations have been done for all of them. It will be shown that there is a clear linear correlation between predicted and measured value. This correlation will be used to analyse the results.

In Figure 18 to 21, the results of the predictions are given for the four materials studied earlier. It is seen that the predicted ear heights are smaller than the measured ones, and that also the amount of earing is underestimated. One possible reason is that the condition $\sigma_{z z}=0$ is too strong. Although in the beginning of the test, the $\sigma_{z z}$ stress component, which is due to the blank holder force, is only a very tiny portion of the flow stress, this is no longer true at the very end of the test. The blank holder force is only carried by the "ears" at the time that the very last parts of the blank are drawn out of the blankholder. They may undergo some additional stretching because of this, at least when there is not enough lubrification. Another possible reason for such additional stretching is that the cups have undergone so called "ironing" during the 


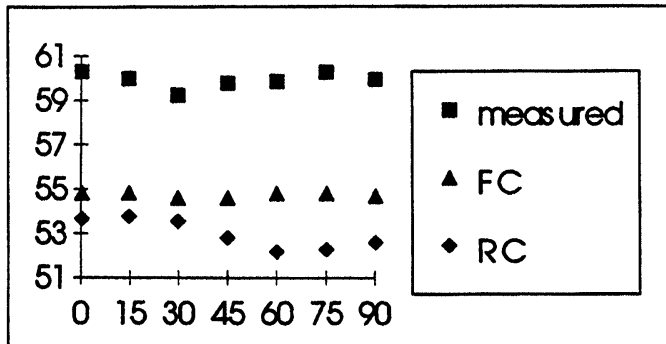

Figure 18 Cup height prediction for material 1.

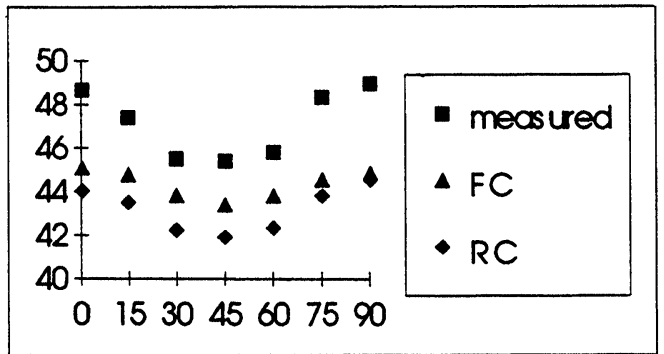

Figure 19 Cup height prediction for material 2.

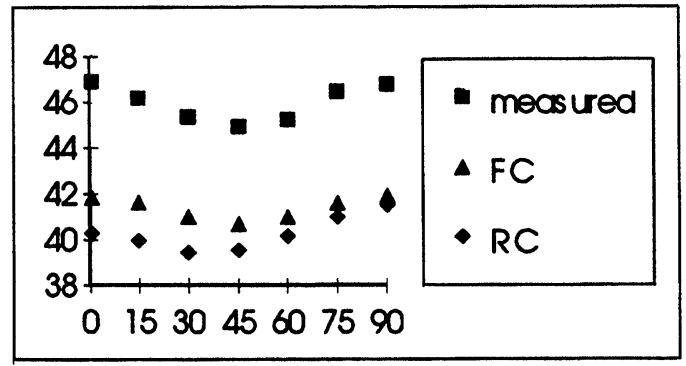

Figure 20 Cup height prediction for material 5.

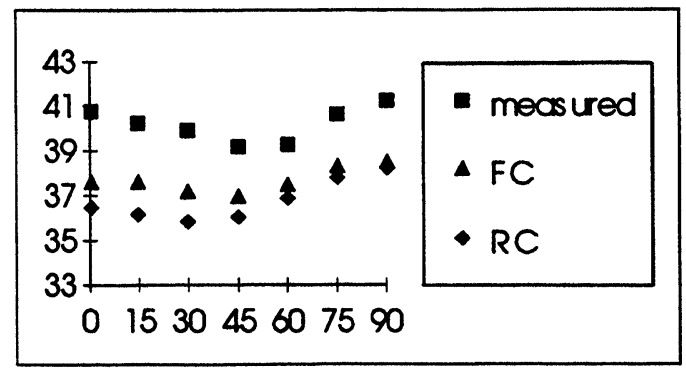

Figure 21 Cup height prediction for material 10. 

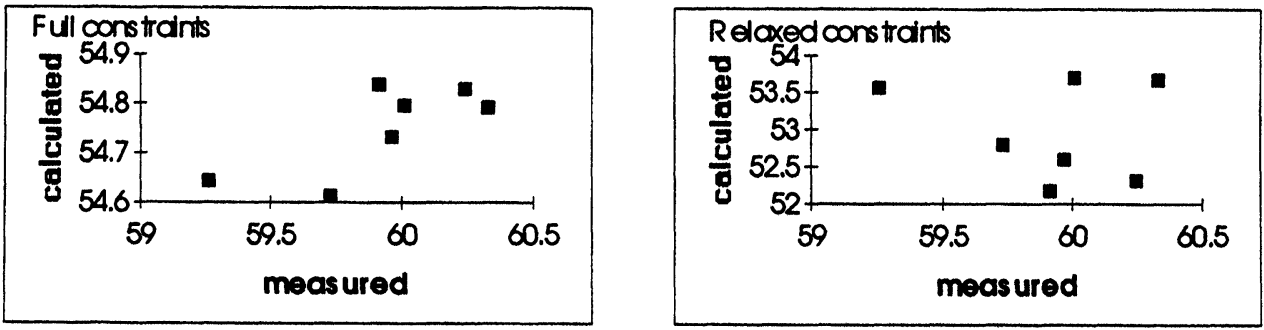

Figure 21 Correlation between measured and predicted value for material 1. Left: full constraints model, right: relaxed constraints.
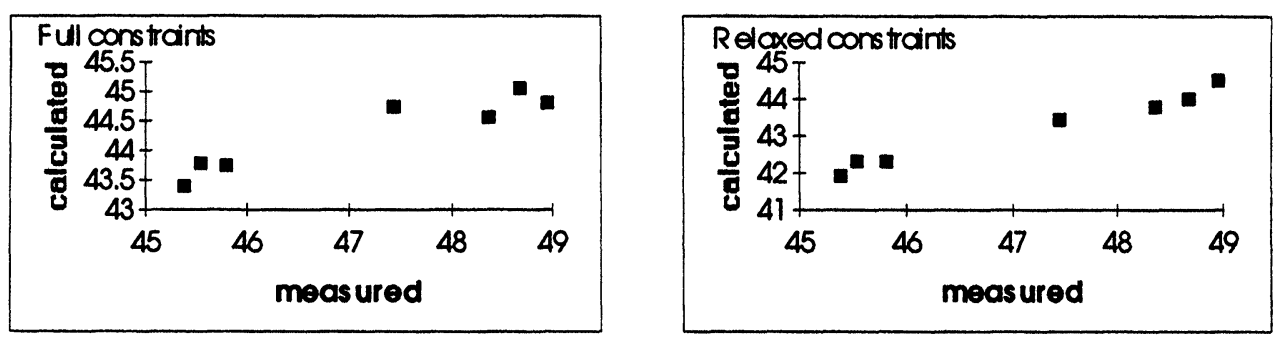

Figure 22 Correlation between measured and predicted value for material 2. Left: full constraints model, right: relaxed constraints.
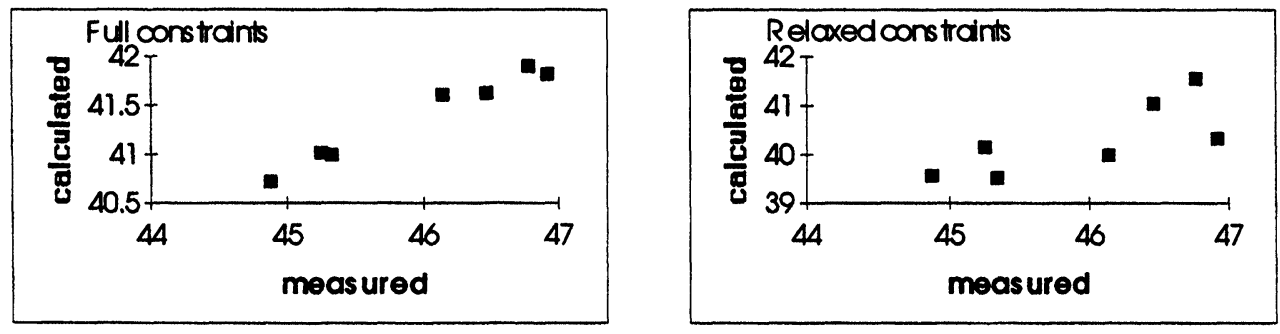

Figure 23 Correlation between measured and predicted value for material 5. Left: full constraints model, right: relaxed constraints.
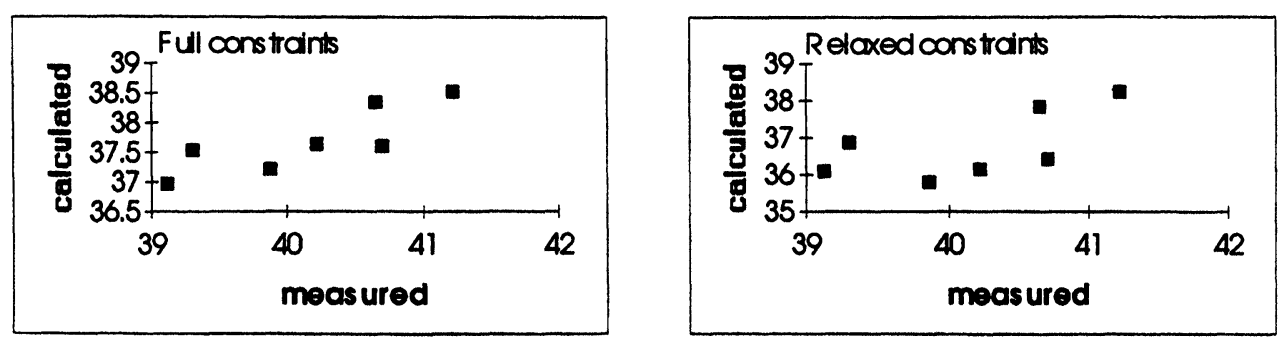

Figure 24 Correlation between measured and predicted value for material 10. Left: full constraints model, right: relaxed constraints. 
deep drawing test. This means that the wall of the cups have undergone an additional elongation due to the tensile stresses occurring there. It can also be observed during the test that the bottom of the cup deforms. Both effects could be successfully simulated by a finite element prediction, using an elasto-plastic model in which an anisotropic yield locus was introduced (Munhoven et al., 1995).

However, one can look at the correlation between the predicted and observed values. In the ideal case, the correlation line between those values would be a line trough the origin with a slope of 1 . It can be seen in Figures 21 to 24 that this is not the case. For the four materials studied earlier, the results for the FC an RC-model are both given.

One can clearly see that for material 1,5 and 10 , the full constraints theory gives the best results. However, in order to make a quantitative evaluation of the results, one should look at the numerical data from a linear regression study which was made for all ten materials. For each of the materials, the result of such a regression is a straight line, characterised by its direction coefficient $\mathrm{m}$ and the intercept on the $y$-axis b. The experimental cup height is then given by:

$$
\text { experimental height }=m \text { (predicted cup height) }+b+\varepsilon
$$

$\varepsilon$ is the residual error. " $s d$ " in Table 2 is an estimate of its standard deviation. In addition, one can determine the coefficient of correlation $\rho$, which gives a measure of the linearity of the relationship between the two variables. This latter is an excellent measure of the accuracy of the correlation, and can be directly compared to the accuracy of the measurements. These 4 parameters are listed in Table 2.

The value of $m$ and $b$ will be mainly dependent on the ironing of the material and the other effects which have been described above, and which are not included in the model. This explains their large. variation. The value of the correlation coefficient $\rho$ gives an impression about in how far the linearity of the relation may be assumed. As a rule of the thumb, one can say that its value has to be larger than 0.9 in order to accept a linear relationship. However, the value of $s d$ is a much more valuable parameter to judge about the accuracy of the regression. Assuming an accuracy on the height measurements of $0.2 \mathrm{~mm}$, and performing a $\chi^{2}$ test, it can be concluded with $99 \%$ confidence that the errors in the approximation are only due to measurement errors on the ear height, if the value of $s$ is smaller than 0.3 .

Table 2 Results of a linear regression study in which the measured values were compared to the predicted values for the FC and RC model, for all ten materials

\begin{tabular}{lcrcccccc}
\hline & \multicolumn{7}{c}{ Full constraints } & \multicolumn{7}{c}{ Relaxed constraints } \\
material & $m$ & $b(\mathrm{~mm})$ & $p$ & $s d(\mathrm{~mm})$ & $m$ & $b(\mathrm{~mm})$ & $p$ & $s d(\mathrm{~mm})$ \\
\hline 1 & 3.0 & -101.3 & 0.74 & 0.3 & -0.1 & 64.3 & 0.15 & 0.4 \\
2 & 2.3 & -53.7 & 0.94 & 0.6 & 1.5 & -18.8 & 0.99 & 0.4 \\
3 & 2.0 & -39.6 & 0.99 & 0.2 & 1.4 & -12.2 & 0.93 & 0.5 \\
4 & 1.8 & -29.7 & 0.97 & 0.3 & 1.3 & -7.8 & 0.94 & 0.4 \\
5 & 1.7 & -24.5 & 0.98 & 0.1 & 0.8 & 13.6 & 0.75 & 0.6 \\
6 & 2.8 & -72.2 & 0.97 & 0.2 & 0.7 & 18.2 & 0.50 & 0.8 \\
7 & 3.7 & -97.9 & 0.97 & 0.3 & 1.5 & -11.1 & 0.97 & 0.3 \\
8 & 3.4 & -102.4 & 0.94 & 0.3 & 1.0 & 2.6 & 0.95 & 0.6 \\
9 & 1.4 & -14.0 & 0.91 & 0.3 & 0.9 & 7.8 & 0.90 & 0.4 \\
10 & 1.1 & -2.6 & 0.83 & 0.5 & 0.5 & 20.7 & 0.64 & 0.6 \\
\hline
\end{tabular}


Only material 2 and 10 do not reach this limit. Nevertheless, for material 10 , the full constraints model is still better than the relaxed constraints model. This does not hold for material 2. For material 7 , both models seem to perform as well.

Looking at the values of $\rho$, it can be concluded that the RC-model gives unsatisfactory predictions for the ear height ( $\rho$ smaller than 0.9 ). This is the case for materials 1 , 5,6 and 10. In contrast to this, the FC model gives only an unsatisfactory correlation for material 1 and 10 .

The position of the ears will be well predicted when the correlation between measured and predicted ear heights is satisfactory as defined above, as is the case for materials 2-9 if the FC model is used. This criterion is however too strict. It is indeed seen in Figure 18 and Figure 21 that even for materials 1 and 10 the FC model predicts the ear positions correctly.

Looking at Table 2, it can also be seen that, except for material 5, the most successful examples have not been chosen for the figures in the previous sections. This latter was done in order to give a clear impression of all possible cases, rather than presenting only the best results. Those are obtained for materials 3 to 7 . It was shown in the previous section that for material 1 , texture evolution should be taken into account. This might also be true for some other materials.

Wagner and Lücke (1994) have also studied the correlation between texture and earing in Al-alloys. There are however some important differences between their study and the present one. First, they did not use a plasticity model such as the TBH model coupled to an analysis of the cup drawing process, but they directly established a linear regression equation between the ear height and the $C$-coefficients of the texture (eq. (2) of their paper). Secondly, this correlation was in principle made separately for each angle $\alpha$ in their notation, which corresponds to the ear positions in our Figure 18-21. As a result, they obtained a data based of correlation coefficients (one set for each angle $\alpha$ ), which is entirely based on experimental observations. Once it has been obtained, it can be used to predict the ear positions (for a certain class of materials, in this case Al-alloys), but it can of course not be used to study the validity of a theoretical model. In our model, the variation of the ear height as a function of the angle ("earing profile") is calculated from the texture by means of the theoretical model without using empirical data. As the shapes of the profile turn out to be correct, but not the absolute values of the maximum and minimum ear heights, the linear regression equation (4) is used, and its coefficients $m$ and $b$ are calculated by fitting to experimental data. For each sample, values for $m$ and $b$ are then found which are valid for all angles for a given sample, but which are different for each material. Wagner and Lücke (1994) find values which are different for each angle, but which are the same for all the materials which they have studied. Still it is remarkable that they have found a very good correlation (Figure $4 \mathrm{a}$ in their paper). In a previous work, one of the present authors indeed also found a good correlation between model predictions and measured percentages of earing for several aluminium alloys (Van Houtte et al., 1987). This may be an indication that factors such as texture development during the cup drawing test itself, ironing, additional stretching of the ears and plastic deformation of the bottom and the wall of the cup, which are not taken into account by the model, introduce less random variability in the case of aluminium alloys than in the case of steels. Another possible source of variability is the fact, that the clearance is not the same for all the samples which have been studied, since they have different thickness and different average $R$-vales. The present authors believe that only a finite element calculation that takes texture into account could take all these factors into account. 
It should finally be noted that for one particular steel, Clarke et al. (1994) also found a good agreement between predicted and measured ear heights if the texture evolution during the cup drawing test was taken into account. This had already been found for aluminium alloys (Van Houtte, 1993) and has also been confirmed by other authors (Ceccaldi et al., 1994).

\section{CONCLUSIONS}

It can be concluded that the relatively simple model which was used for ear height prediction allowed to find the shape of the earing profile of textured materials. This means that for each particular sample, there was a good correlation between the measured and predicted ear heights. The slope of the regression lines were however much larger than unity, which means that the model underestimates the earing tendency. This can be ascribed to the ironing effect, which is not included in the model. It was also found that the regression parameters were different for each material, which is also ascribed to the ironing effect, and to other effects such as plastic deformation in the cup wall and the cup bottom, and the fact that the clearance was not the same for all tests. Effects such as these could be taken into account by a finite element model. Still it is puzzling that for aluminium alloys, previous studies and work by other authors revealed much smaller variations in regression parameters for different samples. It was found that, in contrasts to some literature data, the full constraints Taylor model gave better over-all results than the relaxed constraints version. Indeed the FC model led to a correlation with a standard deviation of the total residual error of less than 0.3 $\mathrm{mm}$ for 9 of the 10 materials, whereas the RC model only did so for 1 of the 10 materials. A possible explanation would be, that the grains of these materials are not very elongated: it has sometimes been argued that $\mathrm{RC}$ models are suited for elongated or flattened grains whereas FC models are suited for equiaxed grains (Van Houtte, 1987).

For some materials, the evolution of the texture during the deep drawing test should be taken into account. This is particularly true for material 1 , in which the 6-ear behaviour is strongly underestimated.

\section{Acknowledgements}

This work has been possible thanks to the financial support of the IWT. The authors wish to thank S. Saimoto and A.P. Clarke for numerous interesting discussions on the subject of earing.

\section{References}

Barlat, F., Panchanadeeswaran, S. and Richmond, O. (1991). Textures and Microstructures, 14-18, 507-512.

Bunge, H. J. (1982). Texture Analysis in Materials Science. London: Butterworths.

Ceccaldi, D., Yala, F., Baudin, T., Penelle, R. and Royer, F. (1994). In Proc. 10th Intntl. Conf. on Textures of Materials (ICOTOM 10), Clausthal, Germany (20-24/9/1993), edited by H.J. Bunge. Materials Science Forum 157-162, 1739-1746.

Clarke, A. P., Van Houtte, P. and Saimoto, S. (1994). In Proc. 10th Intntl. Conf. on Textures of Materials (ICOTOM 10), Clausthal, Germany (20-24/9/1993), edited by H. J. Bunge. Materials Science Forum 157-162, 1953-1960.

Da Costa Viana, C. S., Davies, G. J. and Kallend, J. S. (1978). In Textures of Materials (proc. ICOTOM 5), vol 2, edited by G. Gottstein and K. Lucke, pp. 447-453. Berlin: Springer Verlag.

Daniel, D. and Jonas J. J. (1990). Metall. Trans. 21A, 331-343. 
Daniel, D. Savoie, J. and Jonas J. J. (1993). Acta Metall. Mater., 41, 1907-1920.

Lin, D. W., Daniel, D. and Jonas, J. J. (1991). Mater. Sci. Eng. A131, 161-170.

Munhoven, S., Habraken, A. M., Winters, J., Schouwenaars, R. and Van Houtte, P. (1995). In Simulation of Materials Processing: Theory, Methods and Applications (Proc. Numiform '95, Ithaca, New York 18-21 June 1995), edited by S. F. Shen and P. R. Dawson, pp. 767-772. Rotterdam: Balkema.

Pochettino, A., Pernot, M. and Penelle, R. (1981). In Deformation of Polycrystals, edited by N. Hansen et al., pp. 205-209. Roskilde, Denmark: Risø National Laboratory.

Rodriguez, P. M. B. and Bate, P. S. (1985). In Texture in Non-Ferrous Metals and Alloys, edited by H. D. Merchant and J. G. Morris, pp. 173-187, Warrendale, PA: AIME.

Schouwenaars, R., Van Houtte, P., Aernoudt, E., Standaert, C. and Dilewijns, J. (1994) ISIJ International, 34, 366-372.

Tucker, G. E. G. (1961). Acta Metall. 9, 275-286.

Van Houtte, P. (1984). Textures and Microstructures, 6, 137-162.

Van Houtte, P. (1987). Textures and Microstructures, 7, 29-17.

Van Houtte, P. (1988). Textures and Microstructures, 8-9, 313-350.

Van Houtte, P., Cauwenberg, G., and Aernoudt, E. (1987). Mater. Sci. Eng., 95, 115-124.

Van Houtte, P., Clarke, A. P., Saimoto, S. (1993). In Aluminum Alloys For Packaging, edited by J. G. Morris, E. J. Westerman and P. L. Morris, pp. 261-273. Warrendale, PA: TMS.

Van Houtte, P., Mols, K., Van Bael, A. and Aernoudt, E. (1989). Textures and Microstructures, 11, 23-39.

Wagner, P. and Lücke, K. (1994) In Proc. 10th Intntl. Conf. on Textures of Materials (ICOTOM 10), Clausthal, Germany (20-24/9/1993), edited by H. J. Bunge. Materials Science Forum 157-162, 2043-2048. 